\title{
Semiconductor Magnetoresistive Current Sensor for Weak Current Detection
}

\author{
Qi Zhou*
}

China Special Aero-Vehicle Research Institute of AVIC, Jingmen, Hubei 448000, China

\begin{abstract}
Made up by the combination of InSb-In eutectic magnetoresistive (MR) components, the newly designed semiconductor film magnetoresistive current sensor (MRCS) was to detect weak current with its circuit controlled by signals. This kind of current sensor simultaneously alters the resistance value of two InSb-In magnetoresistive components. Through this way, it could eliminate the coupling capacitance of ultra-large capacity and reduce the volume capacity as well as improve the frequency characteristics. Set a specified parameter through accurate calculation, and make current generate signals which form direct proportion against the magnetic field generated by the weak current of magnetoresistive alteration, reaching the goals of detecting weak current. The test analyzed this current sensor's operating characteristics with the testing pass bands as 7-1800 Hz. It was found that the weak current reached at $10 \mathrm{~mA}$ and could control the effect of temperature drift effectively.
\end{abstract}

\section{KEYWORD}

Magnetoresistive effect

Current sensor

Operating characteristics

\section{Introduction}

Electronic current sensor (MRCS) was made up by InSb-In magnetoresistive components (MR). This kind of current sensor retained the sound performance of Hall current sensor and also had a lot of good features. It had smaller volume, lower cost and simpler structure which made it possible for mass production. The creation of current sensor was to test weak current. This current sensor used the simultaneous alteration of resistance value of two InSb-In magnetoresistive components to achieve this goal. It eliminated the coupling capacitance of ultra-large capacity and reduced volume while improved frequency characteristics.

\section{Characteristics of InSb-In Magnetoresistive Thin Film} The so-called magnetoresistive effect was the corresponding resistance changes along with the changes of increased outer magnetic field. But semiconducting sensitive materials were affected when the direction of electronic current

Copyright () 2015 Qi Zhou

doi: $10.18686 /$ esta.v2i1.5

Received: October 9, 2015; Accepted: November 4, 2015; Published online: December 3

This is an open-access article distributed under the terms of the Creative Commons Attribution Unported License (http://creativecommons.org/ licenses/by-nc/4.0/), which permits unrestricted use, distribution, and reproduction in any medium, provided the original work is properly cited.

${ }^{*}$ Corresponding author: China Special Aero-Vehicle Research Institute of AVIC, Jingmen, Hubei 448000, China. E-mail: zhouqi1120@sina.com was vertical versus the magnetic field which was the function of Lorentz Force. If current carrier changed its direction and led to a longer current travel path, the value of resistance increased. The magnetoresistive effect could be divided into two scenarios: one was physical magnetoresistive effect; the other was geometrical magnetoresistive effect. The physic magnetic field effect would be discussed first. To physic magnetic field, the semiconductor's speed rate had significant differences compared with that of two current carriers (electron and hole). The one with higher speed rate would be the current carrier which caused changes of magnetoresistance. Geometrical magnetoresistive effect was the semiconductor magnetic sensitive resistances with confirmed main materials, and their shapes would be related to magnetoresistive effect. That is to say, their shapes would affect magnetoresistive effects. There were some other magnetoresistances with different ratio of length and width or different shapes; they would have different magnetoresistive effects. In order to increase the geometrical magnetoresistance's effect intensity, various approaches were adopted. The ratio of length and width could be made shorter, for instance the length was several folds of the width; the ratio was less than $1[1,2]$.

\section{Manufacturing Methods of InSb-In Thin Film}

There were many methods to produce InSb-In magnetoresistive components. The most traditional method was to cut the single crystal blocks into thick slices, followed 
by precision work to polish them until they were 30-60 micrometers slices in width. The other one was to create short-circuited electrode. This short-circuited electrode was used to reduce the length and width ratio. Those components required elaborate techniques to use vacuum coating. By mastering the proportion of materials chemometry and crystallization procedure, the indium (In) could form needle shape in the thin slice. Those needles would function as the short-circuited electrode. The main procedure was: substrate polishing; vacuum coating; heat treatment; lithography; preliminary testing and selection; lead meld and measurement for classification. The components were lead, insulation substrate, InSb-In magnetoresistive components MR1 and MR2, permanent magnet, five lead feet etc.

\section{Structure of InSb-In Magnetoresistive Current Sensor}

InSb-In magnetoresistive current sensor consisted of lead, insulation substrate, InSb-In magnetoresistive components MR1 and MR2, permanent magnet and five lead feet. Figure 1 showed the structure of this magnetoresistive current sensor (MRCS). The metal case of InSb-In magnetoresistive current sensor was to shield and protect the sensor. MR1 and MR2 had the same resistance value but were put at opposite positions. The permanent magnet would produce magnetic field B to MR1 and MR2 continuously. The test electric current would go through the lead locating at the axis of symmetry of MR1 and MR2. As shown in Figure 2, MR1 and MR2 were powered by constant current power supply. The electric current was I0. When the testing alternating current went through position 4 and 5 , there would be space magnetic field around the circuit generated. When space magnetic field went through MR1 and MR2 one by one, their directions were opposite at a moment,; therefore, MR1 and MR2 would be affected when this magnetic field overlapped with the partial magnetic field B generated by the permanent magnet of MRCS, and one of the resistance values would increase while the other one would decrease. The changes of resistance values of these two magnetoresistive components would cause changes of electric potentials of $\mathrm{Ua}$ and $\mathrm{Ub}$ in the figure. Amplify the signals of the two ends of $U a$ and $U b$ that entered into differential op-amp circuit t with lead through voltage at different time, we could get to output signal U0 [3].

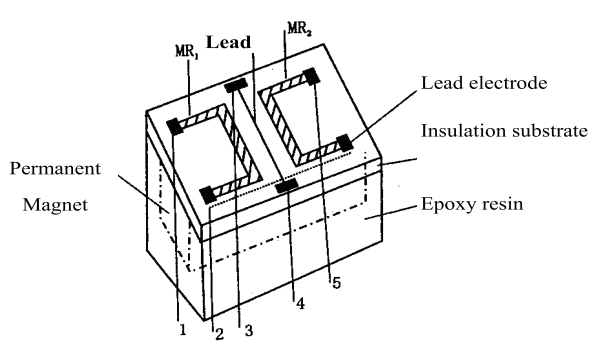

Figure 1. MRCS Structure Diagram.

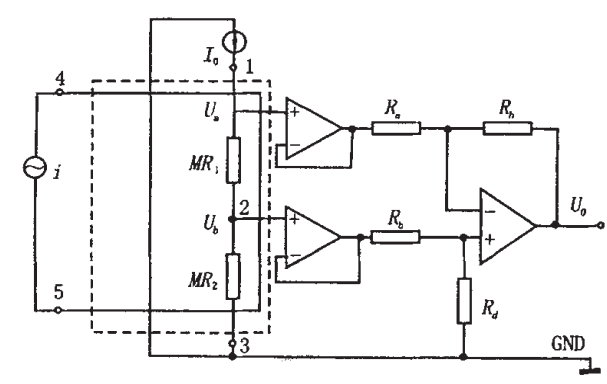

Figure 2. Signal Processing Circuit Diagram.

\section{Discussion}

By maintaining the testing signal at $50 \mathrm{~Hz}$ without fluctuation; increasing the testing current $i$ from $10 \mathrm{~mA}$ to 100 $\mathrm{mA}$; the output voltage would increase from $50 \mathrm{mV}$ to 350 $\mathrm{mV}$ continuously as shown in Figure 3 below.

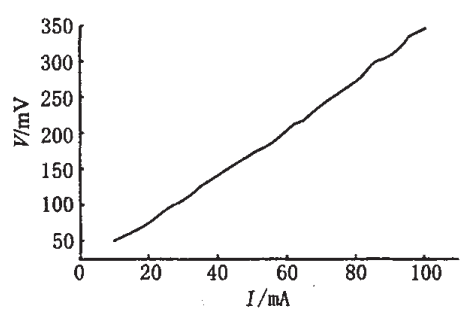

Figure 3. Relation between Output Voltage and Testing Current.

By maintaining the testing signal at $60 \mathrm{~mA}$ without fluctuation, the frequency would increase from $1 \mathrm{~Hz}$ to 20,000 $\mathrm{Hz}$. When the monitored frequency was $70-500 \mathrm{~Hz}$, the output voltage was the max as $208 \mathrm{mV}$; however when the frequency was $1-70 \mathrm{~Hz}$, the output voltage increased gradually and slowly; when the frequency was $500-15,000 \mathrm{~Hz}$, the output voltage decreased slowly; when frequency was above $15,000 \mathrm{~Hz}$, the output voltage did not change a lot but at a stable level. Therefore, as shown in Figure 4, with passband within $7-1,800 \mathrm{~Hz}$, the output values reached the detecting goal.

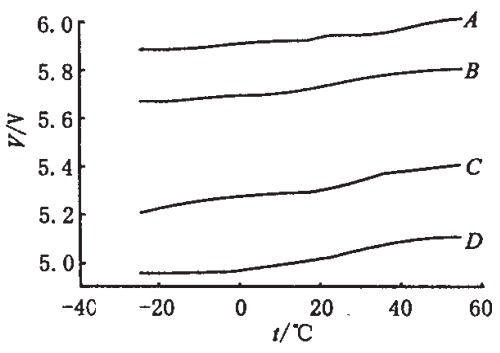

Figure 4. Relation of Output Voltage and Temperature.

The relation between the current and surrounding space magnetic field illustrated that when straight line went through alternating current, the result could be achieved through calculation that U0 would increase 
continuously and linearly along with the increase of $i$ as shown in Figure 3.

However, when the circuit in static operating status while raising the external temperature from -25 to $55^{\circ} \mathrm{C}$, the output voltages of Sample A, B, C and D did not vary significantly. All of the four curves indicated a trend of increase as shown in Figure 4.

Changes of temperature would cause some changes of magnetoresistance of both InSb. By calculating through formula, the result would be $\mathrm{U} 0=0$.

The experiments indicated clearly that this design could theoretically control the effect of temperature drift effectively. According to Figure 4, the four curves illustrated the results of the four samples $\mathrm{A}, \mathrm{B}, \mathrm{C}$ and $\mathrm{D}$ who shared the same circuits' structures. Their static working performances were different; this would be related with actual existing gap between the same components. When the temperature increased from -25 to $55^{\circ} \mathrm{C}$, the output voltage changing scope was $0.14-1.21 \mathrm{~V}$; the changing rate was $0.0294 \%-0.0491 \%$ per Celsius. It was associated with the performance changes of components along with temperature changes $[4,5]$.

\section{Conclusion}

We created a signal processing circuit which could create possible large output signals by using InSb-In eutectic typed thin film magnetoresistive components, bias magnet and magnetoresistive current sensor put at the axle wire of circuit. This experiment indicated that there were connections related to changes among components. When the testing signal's current intensity or frequency changed, the magnetic field of the InSb magnetoresistance would change correspondingly. The value of resistance would change, leading to the changes of input signals $\mathrm{Ua}$ and $\mathrm{Ub}$.

Different modules management of the input signals could be adopted to figure out the most suitable circuit parameters. Remove the sideling straight line current level. Finally, the amplified signal with direct proportion against the testing weak current could be obtained.

The transmission band of this current sensor was 7-1,800 $\mathrm{Hz}$; the detected weak current signal was $10 \mathrm{~mA}$; it had strong inhibiting effect on temperature drift.

\section{References}

1. Zhai, X. M. (2012). Research of High Precision Detection of Transmission Line Insulator Leakage Current and Data Compression Technology. North China Electric Power University.

2. Xing, J. (2010). Non-contact Weak Current Detection System Based on System Proportion Measurement and Application. Tianjin University.

3. Cheng, Z. Q. (1988). 'Magnetic Sensor and Application' Lecture; Lecture Four: Magnetic Sensitive Resistance Sensor I. Automation Instrumentation, 08, 39-44.

4. Zhou, D. Y., \& Huang, Z. H. (2004). Design of Indium Antimonide Gear Sensor in Measurement of Low Rotating Speed. Computer Measurement and Control, 09, 898900.

5. Huang, D. X. (1990). Sensitive Technical Physics and Application Series [IV] Magnetic Sensitive Sensor and Application. Physics, 07, 409-413. 